\title{
OBITUARY
}

\section{Donald Charles Backer (1943-2010)}

\author{
Astronomer who discovered a new class of pulsar.
}

Radio astronomy has generally progressed as a result of the combined talents of engineers who build detector electronics, project leaders who organize the building and sharing of radio telescopes, and scientists with a nose for identifying ventures likely to yield great discoveries. Unusually skilled in all these areas, Donald Backer discovered millisecond pulsars, and was among the first to image the radio-wave emission from the giant black hole at the centre of our Galaxy.

Backer, who died on 25 July, was born in Plainfield, New Jersey, in 1943. He received a bachelor's degree in engineering physics from Cornell University in Ithaca, New York, in 1966. He then completed an MSc in radio astronomy at the University of Manchester, UK, before returning to Cornell to pursue a $\mathrm{PhD}$.

Backer's $\mathrm{PhD}$ years coincided with one of the most exciting periods in astronomy. The Arecibo telescope in Puerto Rico, still the world's largest single-dish radio telescope, was only a few years old. And in 1967, pulsars were discovered - magnetized and spinning, dense remnants of massive stars that emit beams of electromagnetic radiation.

The first pulsars discovered emitted regular bursts of radiation every second or so, but the amount of radiation astronomers detected in each pulse varied seemingly erratically. Part of the explanation turned out to lie with factors extrinsic to the pulsars: just as visible light from stars twinkles as it propagates through Earth's turbulent atmosphere, so radio waves from pulsars twinkle or 'scintillate' as they travel through the interstellar medium. Backer explored this pulse-to-pulse variation for his $\mathrm{PhD}$; his most notable finding was the discovery of 'nulling' pulsars that occasionally (and mysteriously) 'forget' to pulse.

While a postdoctoral fellow at the National Radio Astronomy Observatory (NRAO) in Charlottesville, Virginia, and later at the Goddard Space Flight Center in Greenbelt, Maryland, Backer explored the use of interferometry including very-long-baseline interferometry (VLBI) to essentially 'zoom in' on astronomical radio-wave sources. This technique involves linking telescopes spaced far apart across countries, continents and even the globe, to produce highresolution images. Backer initially focused on measuring the motion of pulsars and investigating the nature of Sagittarius $\mathrm{A}^{\star}-\mathrm{a}$ source of radio waves associated with the

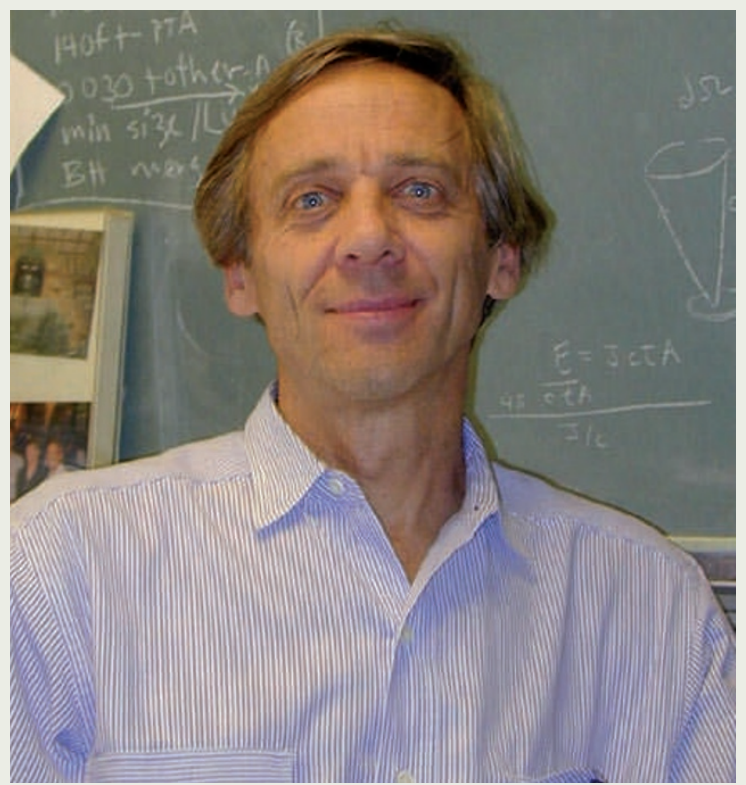

abandon their companion stars. But frenzied worldwide searches motivated by its discovery uncovered many other pulsars still spinning around companion stars, and even pulsars in 'globular clusters' - the most ancient star collections in our Galaxy.

The reliability of the pulses of radiation emitted by PSR $1937+21$ made it a very stable 'clock'. Indeed, since the discovery of millisecond pulsars, astronomers including Backer have used them to try to detect the gravitational-wave signals predicted to have accompanied the birth of the Universe and mergers of giant black holes. (Because gravitational waves displace the position of Earth in relation to the pulsars, they can be detected by shifts in pulse arrival times.)

As Backer rose through the ranks

black hole at the centre of our Galaxy. Backer and Richard Sramek, who was also at the NRAO (where he works still), were able to track the path of the Sun around our Galaxy. They were also able to calculate that the mass of the Milky Way's black hole is more than a few hundred thousand times that of the Sun. Decades later, Backer and his group managed to further erode the blurriness of Sagittarius A*, caused by the turbulent interstellar medium, and determine its physical size.

In 1975, Backer joined the Radio Astronomy Laboratory of the University of California, Berkeley, where he promoted VLBI both locally and internationally. A few years earlier, astronomers at the University of Cambridge, UK, had identified a peculiar source of radio waves in the Fourth Cambridge catalogue of radio sources.

Denoted as 4C21.53, it displayed properties characteristic of pulsars, but had not been detected by pulsar surveys at Arecibo and elsewhere. Backer's determination to solve the mystery led him and his group to find, in 1982 , that $4 \mathrm{C} 21.53$ was a pulsar rotating at the unprecedented rate of once every 1.56 milliseconds. The object was named PSR 1937+21 — the first millisecond pulsar to be discovered.

This finding of a new class of star that rotates thousands of times each second was a sensation. Other astronomers quickly suggested that such pulsars are the end states of neutron stars accreting matter from a companion star. It turns out that PSR $1937+21$ is one of several rare pulsars that have somehow managed to destroy or at Berkeley (he became director of the Radio Astronomy Laboratory in 2008), he relentlessly pursued the idea of a 'pulsar timing array' - a network of radio telescopes for observing widely separated pulsars - to look for gravitational waves. To this end, he co-founded the Center for Astronomy Signal Processing and Electronics Research (CASPER) at Berkeley in 2004. This programme aims to encourage collaborative designs for pulsar radio-signal detection and processing, as well as for interferometry applied to celestial objects in general.

Don had a gift for homing in on the fundamental problems in astronomy, thought innovatively about instrumentation, and was a natural leader owing to his gentle manners, modesty and concern for others. In recognition of his pioneering contributions to observational astronomy, he received the Jansky prize in 2003, one of the highest achievements for a radio astronomer. At the time of his death, he had three major scientific goals: to image Sagittarius $\mathrm{A}^{\star}$ in the millimetre wavelength band and so peer close to the event horizon (edge) of our Galaxy's central black hole; to explore the ionization of hydrogen pervading the early Universe; and to detect the gravitational signatures of merging supermassive black holes. In all these areas, answers are likely to arrive within the next decade. When they do, all involved will owe a debt of gratitude to Don Backer.

\section{S. R. Kulkarni}

S. R. Kulkarni is at the California Institute of Technology, Pasadena, California 91125, USA. e-mail: srk@astro.caltech.edu 